\title{
Pathogenic Variability of Isolates of Rhizoctonia solani of Different Agricultural Crops
}

\author{
Manashi Debbarma* and Pranab Dutta \\ School of Crop Protection, College of Post Graduate Studies in Agricultural Sciences, Central \\ Agricultural University (Umiam), Meghalaya, India \\ *Corresponding author
}

Keywords

Pathogenicity, Host, Rhizoctoniasolani

Article Info

Accepted:

12 December 2020

Available Online:

10 January 2021
Rhizoctonia solani is an ubiquitous soil borne plant pathogen causes versatile diseases in agricultural crops. Due to the attack of the pathogen, plant show symptom like damping off, wire stem, stem rot, collar rot, web blight, charcoal rot, black scurf, sheath blight and banded leaf and sheath blight etc in different host crops. In the present study we isolated different isolates of $R$. solani from different host plant of different locations. The association of pathogenic isolates was confirmed by Koch postulate which was conducted on hypocotyl by mixing the soil with culture having $R$. solani and seeds were sown for germination. Fifteen days after the germination of seeds, seedlings showed symptoms, whereas controlled seedlings remained healthy. Further, pathogenicity test was also conducted on leaves by inoculating freshly cultured mycelial disc $(5 \mathrm{~mm})$ on healthy plants of different hosts, non-inoculated plants served as control. Few inoculated plants developed symptoms, whereas controlled plants remained healthy. We also evaluated cross infectivity of different isolates in different crops plant through standard method and found that isolates of rice (RS-1 and RS-2) and setaria (RS-4) causes infection to all the tested host plants like maize, green gram, cabbage and potato being highest score 5 . But few isolates of $R$. solani, RS-3, RS-5, RS-5, RS-7, RS-8, RS-9, RS-10 could not cause infection to all the host plant tested except few. Long pepper (RS-6) could not cause any infection on maize and green gram and showed score 0 .

\section{Introduction}

\section{Rhizoctonia}

solani

Thanatephorus spp.) is soil borne plant pathogenic fungus, which is anubiquitous in nature with a wide host range and worldwide distribution causing diseases ranging from field to horticultural crops (Snehet al., 1996). $R$. solani frequently exists as thread-like growth on plants or in culture, and is considered as a soil borne pathogen. The infection caused by $R$. solanican attack the crop in any stage. viz., from seedling to harvesting stage. It is best known to cause various plant diseases such as collar rot (Dutta and Das, 2002), root rot, damping off, wire stem, stem rot (Dutta and Das, 1999), bare patch of cereals, root rot of sugar beet, belly rot of cucumber, sheath blight of rice (Das et al., 1997), black scurf of potatoes. 
Rhizoctonia can be found across all areas of the world (environmental conditions permitting) where its host crops are located.

R. solani shows high variability in its cultural characters and pathogenic behavior. The variability is so high that it is difficult to classify different isolates into groups. Isolates of multinucleate $R$. solani are genetically diverse in their cultural, morphological and physiological characteristics as well as in their pathogenic range of host plants (Kuninga et al., 1997; Gonzalez et al., 2001 and 2006; Sharon et al., 2006 and 2008). But literature related to the isolates of north eastern states of India, their variability of infection is rare. Searching literature also showed that cross infectivity test of Rhizoctonia isolates of different host of the region has not been done.

With this background, the present study was conducted to isolate the different isolates of $R$. solani from different crop host and studied variability on pathogenic ability through cross infectivity tests.

\section{Materials and Methods}

The experiment was conducted in the net house of Mycology Research Section, Department of Plant Pathology, Assam Agricultural University (AAU), Jorhat, Assam.

\section{Collection of sample}

Diseased samples of different crops showing typical symptom infected by $R$. solani were collected from Assam (Instructional Cum Research farm and Experimental farm, Department of Horticulture, AAU, Jorhat) in air tight zip plastic bag. Collected samples were brought to the laboratory of Mycology Research Section, AAU, Jorhat, Assam.

\section{Isolation and purification of putative} organism

To isolate the fungi, collected diseased samples were surface sterilized with 4.0 percent sodium hypochlorite $(\mathrm{NaOCl})$ solution and then rinsed twice with double distilled sterile water. In sterile Petriplates, the diseased specimens were crushed and a small portion (size: 1-2mm) of infected part with small healthy parts were transferred to culture plate containing PDA under aseptic condition. Inoculated plates were incubated at $25 \pm 1^{\circ} \mathrm{C}$ for 5-7 days.

Plates were observed regularly for growth and development of associated micro-organisms. After five days of incubation, the organisms were sub-cultured for purification by selecting the desired colonies. Pure culture of each isolate was made by transferring them to fresh PDA plates following the technique of hyphal tip culture method.

\section{Maintenance of culture}

Isolated and purified cultures were maintained by transferring periodically in fresh PDA medium and storing in refrigerator at $4^{\circ} \mathrm{C}$ for further studies.

\section{Preparation pot mixture and planting}

Inoculum for infestation of soil was prepared by mixing seven (7) days old culture of $R$. solani on Potato Dextrose Agar (PDA) media $(100 \mathrm{ml})$ in $100 \mathrm{ml}$ of sterile water and pots were filled with the infested soil. Suspension of fungal propagules were thoroughly mixed with soil @ 4.0 per cent on dry weight equivalent.

The infested soil was kept moist for three (3) days before planting. Ten seeds were sown in each of three (3) replicate pots of infested soil. 


\section{Pathogenicity test}

Pathogenicity test of the isolated, purified and characterized isolates of $R$. solani were conducted between crops. Inoculum of the pathogen more particularly mycelium were inoculated on both hypocotyls and leaves of the targeted plants. Each plant was grown individually in pre-sterilized soil in earthen and plastic pots and arranged in a completely randomized block design in a greenhouse.

\section{Pathogenicity test on hypocotyls}

Pathogenicity of ten (10)collected isolates of $R$. solani were carried out in pots filled with sterilized soil. Healthy seedlings of potato, rice, green gram, cabbage and maize were selected for inoculation 1 week after seeding. Inoculum for infestation of soil was prepared by mixing seven (7) days old culture on Potato Dextrose Agar (PDA) media (100 ml) in $100 \mathrm{ml}$ of sterile water. Suspension of fungal propagules were thoroughly mixed with soil @ 4 per cent on dry weight equivalent. The infested soil was kept moist for three (3) days before planting. Ten seeds were sown in each of three (3) replicate pots of infested soil. Seedlings grown in sterilized soil without inoculum served as control. Disease incidence was recorded after fifteen (15) days of inoculation. Disease severity was recorded on six(6) point scale given by Zhou et al., 2009.

0: Seedling well- developed, no visible lesions on lower stem and roots 1: Seedling growing well with a few small lesions on lower stem and roots, total infected area $<25$ per cent.

2: Growth of the seedling is retarded, moderate necrosis or small lesions scattered over the lower stem and roots, total infected area $25-50$ per cent.

3: Seedling growth is hampered, large lesions on the lower stem and roots, total area 50-75 per cent.

4: Seedling growth is seriously hampered, girdling lesions on the lower stem and roots, total infected area $>75$ per cent.

5: Death of plant. Treatment combinations followed to test the pathogenicity of ten different isolates of $R$. solani on hypocotyls of five different crops were as follows

\begin{tabular}{|c|c|}
\hline $\begin{array}{c}\text { T }_{1}: \text { RS-1 + Green gram } \\
\text { T}_{2}: \text { RS-1 + Maize } \\
\text { T }_{3}: \text { RS-1 + Cabbage } \\
\text { T }_{4}: \text { RS-1 + Potato } \\
\text { T }_{5}: \text { RS-2 + Green gram } \\
\text { T }_{6}: \text { RS-2 + Maize } \\
\text { T }_{7}: \text { RS-2 + Cabbage } \\
\text { T}_{8}: \text { RS-2 + Potato } \\
\text { T }_{9}: \text { RS-3 + Green gram } \\
\text { T }_{10}: \text { RS-3 + Maize } \\
\text { T }_{11}: \text { RS-3 + Cabbage }\end{array}$ & $\begin{array}{c}\text { T }_{21}: \text { RS-6 + Green gram } \\
\text { T } 22: \text { RS-6+ Maize } \\
\text { T } 23_{23}: \text { RS-6 + Cabbage } \\
\text { T }_{24}: \text { RS-6 + Potato } \\
\text { T } 25: \text { RS-7 + Green gram } \text { T }_{26}: \text { RS-7 + Maize } \\
\text { T }_{27}: \text { RS-7 + Cabbage } \\
\text { T }_{28}: \text { RS-7 + Potato } \\
\text { T }_{29}: \text { RS-8 + Green gram } \\
\text { T }_{30}: \text { RS-8 + Maize } \\
\text { T }_{31}: \text { RS-8 + Cabbage }\end{array}$ \\
\hline
\end{tabular}




\begin{tabular}{|c|c|}
\hline $\mathrm{T}_{12}: \mathrm{RS}-3$ + Potato & $\mathrm{T}_{32}: \mathbf{R S - 8}+$ Potato \\
\hline T $_{13}:$ RS-4 + Green gram & T $_{33}:$ RS-9 + Green gram \\
\hline T $_{14}:$ RS-4 + Maize & T $_{34}:$ RS-9 + Maize \\
\hline $\mathrm{T}_{15}: \mathrm{RS}-4$ + Cabbage & T $_{35}:$ RS-9 + Cabbage \\
\hline $\mathrm{T}_{16}: \mathrm{RS}-4$ + Potato & $\mathbf{T}_{36}: \mathbf{R S}-9$ + Potato \\
\hline $\mathrm{T}_{17}: \mathrm{RS}-5$ + Green gram & $\mathrm{T}_{37}: \mathbf{R S}-10$ + Green gram \\
\hline $\mathbf{T}_{18}: \mathbf{R S}-5+$ Maize & $T_{38}:$ RS-10 + Maize \\
\hline T $_{19}:$ RS-5 + Cabbage & $\mathbf{T}_{39}:$ RS10 + Cabbage \\
\hline $\mathrm{T}_{20}:$ RS-5 + Potato & $\mathbf{T}_{40}: \mathbf{R S}-10+$ Potato \\
\hline
\end{tabular}

\section{Pathogenicity test on leaves}

Pathogenicity of $R$. solani was determined by inoculating the leaves of 30 days old seedlings growing in previously steamed soil. Inoculation was done by placing a mycelial agar disc $(6 \mathrm{~mm} \mathrm{dia.})$ in the center of each leaf. After inoculation, plants were covered with a plastic bag for $24 \mathrm{hr}$. PDA agar disc(6 $\mathrm{mm}$ dia.) without fungus was served as control. For each isolates three plants per pot were inoculated and replicated thrice. Five days after inoculation, the severity of leaf infection was rated on six point scale as mentioned above.

Treatment combinations followed to test the pathogenicity of ten (10) different isolates of $R$. solani on leaves of five (5) different crops were same as pathogenicity test on hypocotyls as mentioned above.

\section{Results and Discussion}

\section{Cultural characteristics of $R$. solani}

Colony colour, growth pattern and radial growth of ten different isolates of $R$. solani were observed. Colony colour, growth pattern and radial growth showed great diversity in all the isolates. Based on colony pigmentation, isolates were classified as white, grey, yellow and orange. Radial growth (diameter) of $R$. solani was recorded after 72 hrs of incubation. Based on radial growth pattern, all the Rhizoctonia isolates were classified into three groups - abundant, moderate, and scarce.

\section{Morphological characteristics of different isolates of $R$. solani}

For morphological characters, branching pattern, septa, width of hypha and nuclei number of $R$. solani were studied on Compound microscope. Compound microscope studies revealed that all the isolates of $R$. solani characteristically having hyphal branching at right angle, septate with lateral branches constricted at the junction. Hyphal width of all the isolates of $R$. solani ranged from $5.6 \mu \mathrm{m}$ to $7.2 \mu \mathrm{m}$.

Pathogenicity test of different isolates of $\boldsymbol{R}$. solani

\section{Pathogenicity test on hypocotyls}

Results of cross inoculation of inoculum of all the isolates of $R$. solani (Plate 1and 2) showed positive reaction to the hypocotyl of potato with severity score 4 (seedling growth is hampered, large lesions on the lower stem and roots). Two isolate (RS-1 and RS-2) cause 
infection on all the five tested crops (rice, maize, green gram, cabbage and potato). Highest disease severity score of 5 was recorded by RS-1, RS-2, RS-3, RS-4, RS-5, RS-6, RS-9 and RS-10 on cabbage (Plate 3b, Table 1). All the infected seedlings were found dead after 15 days of its germination. Isolate RS-3, RS-5, RS-6, RS-9 and RS-10 could not able cause infection on hypocotyls of maize showing disease severity score of 0 i.e., seedling well developed, no visible lesions on lower roots developed (Plate $3 b$ ). Similarly, RS-6 to RS-10 did not cause infection on green gram but RS-1, RS-2, RS3 , RS-4 and RS-5 cause severe infection with disease severity score of 5 causing total death of the seedlings (Plate 3a). All the ten isolates of $R$. solani cause infection on hypocotyl of potato with disease severity score of 4 . Whereas in case of rice all the ten isolates though cause hypocotyls infection but with variable disease score. RS-1, RS-2, RS-3, RS4, RS-7 and RS-8 cause disease severity score of 4, isolate RS-5 and RS-6 cause disease score of 3 and RS-9 and RS-10 cause disease severity score of 1 (Table 1 ).

Table1 Reaction and disease severity of different isolates of Rhizoctonia solani on hypocotyl of different hosts

\begin{tabular}{|l|c|c|c|c|c|c|}
\hline \multicolumn{1}{|c|}{ Sources } & Isolates & Rice & Maize & Green gram & Cabbage & Potato \\
\hline Rice(Sheath) & RS-1 & +ve (4)* & +ve (4) & +ve (5) & +ve (5) & +ve (4) \\
\hline Rice(Leaf) & RS-2 & +ve (4) & +ve (4) & +ve (5) & +ve (5) & +ve (4) \\
\hline Cowpea(Collar) & RS-3 & +ve (4) & -ve (0) & +ve (5) & +ve (5) & +ve (4) \\
\hline Setaria & RS-4 & +ve (4) & +ve (4) & +ve (5) & +ve (5) & +ve (4) \\
\hline Greengram & RS-5 & +ve (3) & -ve (0) & +ve (5) & +ve (5) & +ve (4) \\
\hline Long pepper & RS-6 & +ve (3) & -ve (0) & -ve (0) & +ve (5) & +ve (4) \\
\hline Maize(Leaf) & RS-7 & +ve (4) & +ve (4) & -ve (0) & -ve (0) & +ve (4) \\
\hline Maize-T & RS-8 & +ve (4) & +ve (4) & -ve (0) & -ve (0) & +ve (4) \\
\hline Cauliflower-T & RS-9 & +ve (1) & -ve (0) & -ve (0) & +ve (5) & +ve (5) \\
\hline Cabbage-T & RS-10 & +ve (1) & -ve (0) & -ve (0) & +ve (5) & +ve (4) \\
\hline
\end{tabular}

*Data are mean of three replications and data in the parentheses are disease severity score.

$(+\mathrm{ve}=$ Infection occurred, - ve $=$ No infection observed $)$

Table.2 Reaction and disease severity of different isolates of Rhizoctonia solani on leaves of different hosts

\begin{tabular}{|c|c|c|c|c|c|c|}
\hline Source & Isolates & Rice & Maize & Green gram & Cabbage & Potato \\
\hline Rice-J & RS-1 & $+\operatorname{ve}(4) *$ & +ve (3) & +ve (3) & +ve (5) & -ve (0) \\
\hline Rice-T & RS-2 & +ve (4) & +ve (3) & +ve (3) & $+v e(5)$ & -ve (0) \\
\hline Cowpea(Collar) & RS-3 & $+\mathrm{ve}(3)$ & $+\mathrm{ve}(2)$ & +ve (2) & $+v e(5)$ & -ve (0) \\
\hline Setaria & RS-4 & +ve (3) & -ve (0) & +ve (3) & +ve (5) & +ve (3) \\
\hline Greengram & RS-5 & +ve (4) & -ve (0) & $+v e(5)$ & $+\mathrm{ve}(5)$ & $+v e(3)$ \\
\hline Long pepper & RS-6 & +ve $(0)$ & -ve (0) & +ve (3) & $+v e(5)$ & +ve (3) \\
\hline Maize-J & RS-7 & +ve (4) & +ve (4) & +ve (3) & $+v e(5)$ & -ve (0) \\
\hline Maize-T & RS-8 & +ve (4) & $+\mathrm{ve}(4)$ & +ve (3) & $+v e(5)$ & -ve (0) \\
\hline Cauliflower-T & $\mathrm{RS}-9$ & -ve (0) & -ve (0) & +ve (3) & $+v e(5)$ & -ve (0) \\
\hline Cabbage-T & RS-10 & -ve (0) & -ve (0) & +ve (3) & +ve (5) & -ve (0) \\
\hline
\end{tabular}

*Data are mean of three replications and data in the parentheses are disease severity score $(+\mathrm{ve}=$ Infection occurred, - ve $=$ No infection observed $)$ 
Plate.1 (a-f) Preparation of inoculum of different isolates of R. solani

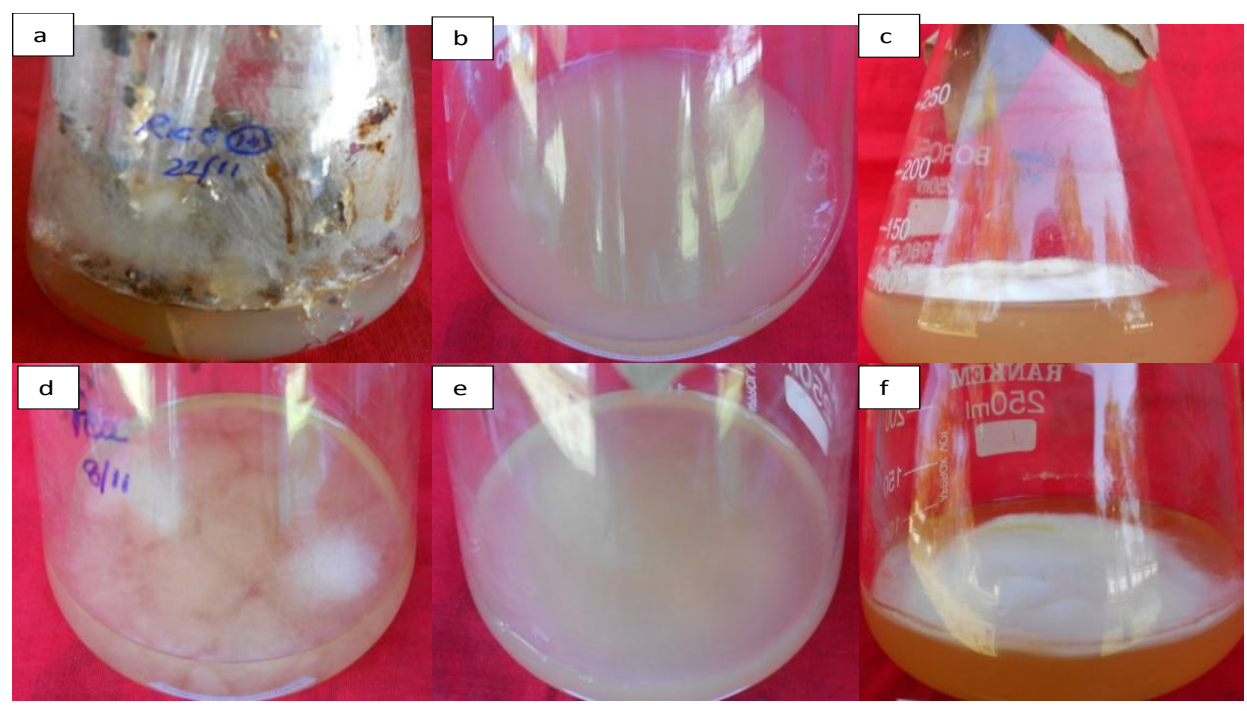

(a: RS-1, b: RS-2, c:RS-3, d:RS-4, e:RS-5, f:RS-6)

Plate. 2(a-e) General view of pot experiment for cross inoculation with different isolates of $R$. solani on different host
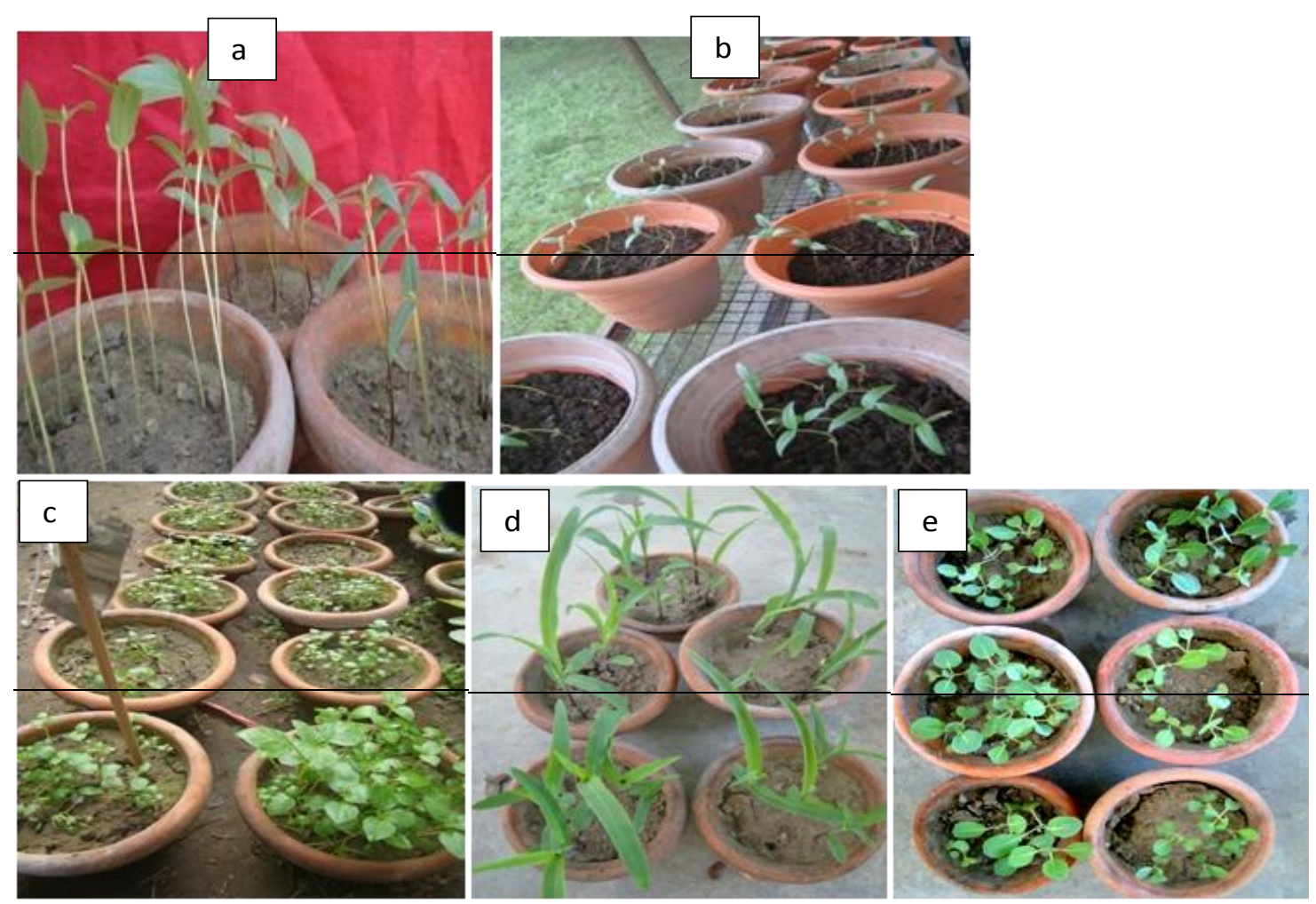

a: Green gram b: Green gram, c: Potato, d: Maize, e: Cabbage 
Plate.3(a-c) Cross inoculation of different isolates of $R$. solani on
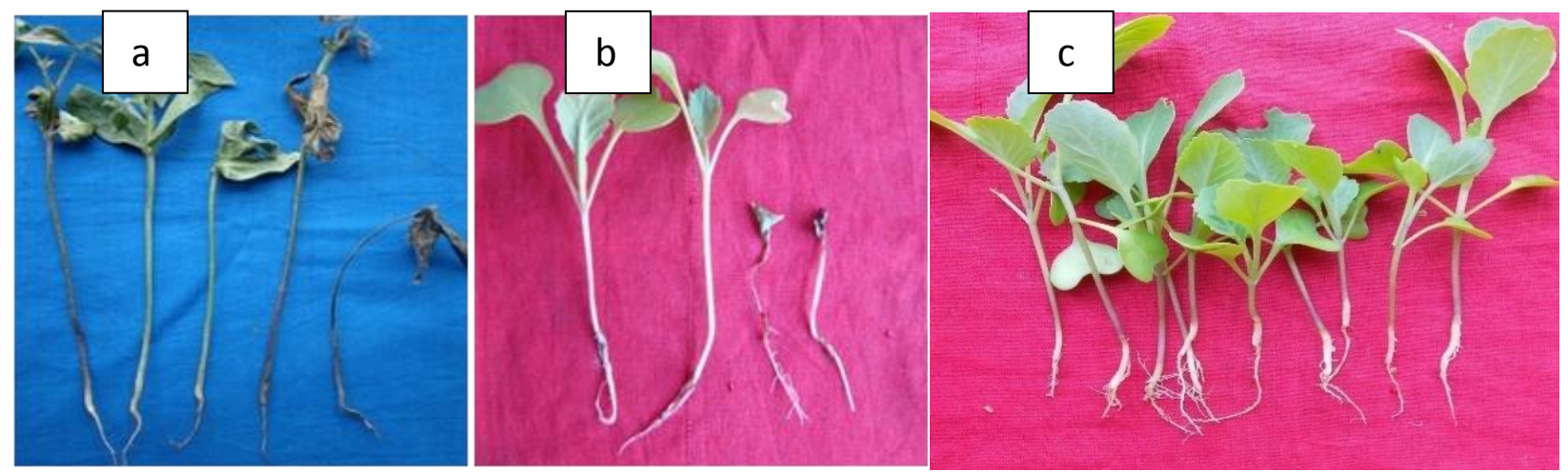

a. Green gram b. Cabbage showing the symptom c. Healthy cabbage seedling in control

Plate.4 (a-h) Cross inoculation of different isolates of $R$. solani on different crops
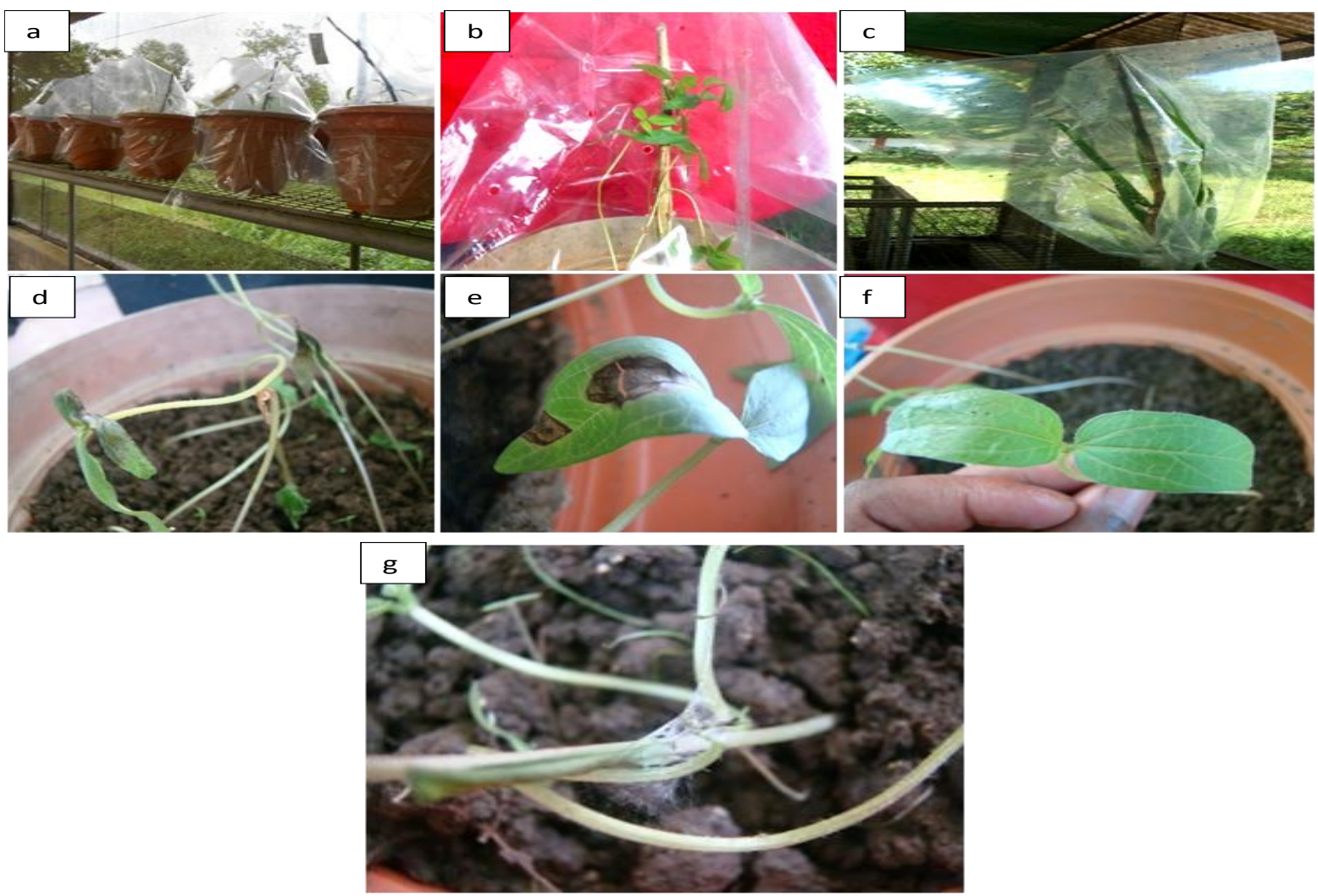

a. General view of inoculated plant

b. Closer view of inoculated plant covered with polybag for maintenance of humidity

c. Inoculated leaves of rice covered with polybag for maintenance of moisture

d. Infection on green gram leaf by RS-1

e. Healthy green gram leaf after inoculation with RS-8

f. Infection on green gam leaf by RS-3

g. Infection on green gram leaf by RS-4 
Plate.5 (a-d) Reactions of different isolates of $R$. solani on inoculation to rice seedlings
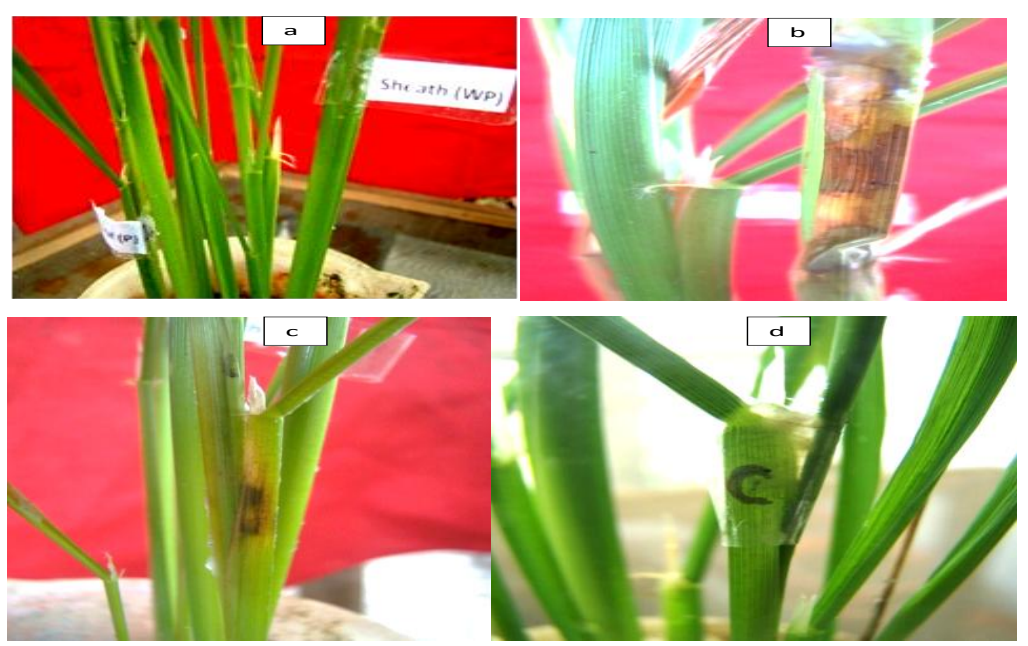

a: Inoculated (and marked) leaf and sheath of rice hill

b: Rice seedling showing symptom of infection of RS-8

c: Infection of RS-5 on rice

d: Control (no infection)

Plate.6 (a-g) Reactions of different isolates of $R$. solani on inoculation to potato leaves and seedlings
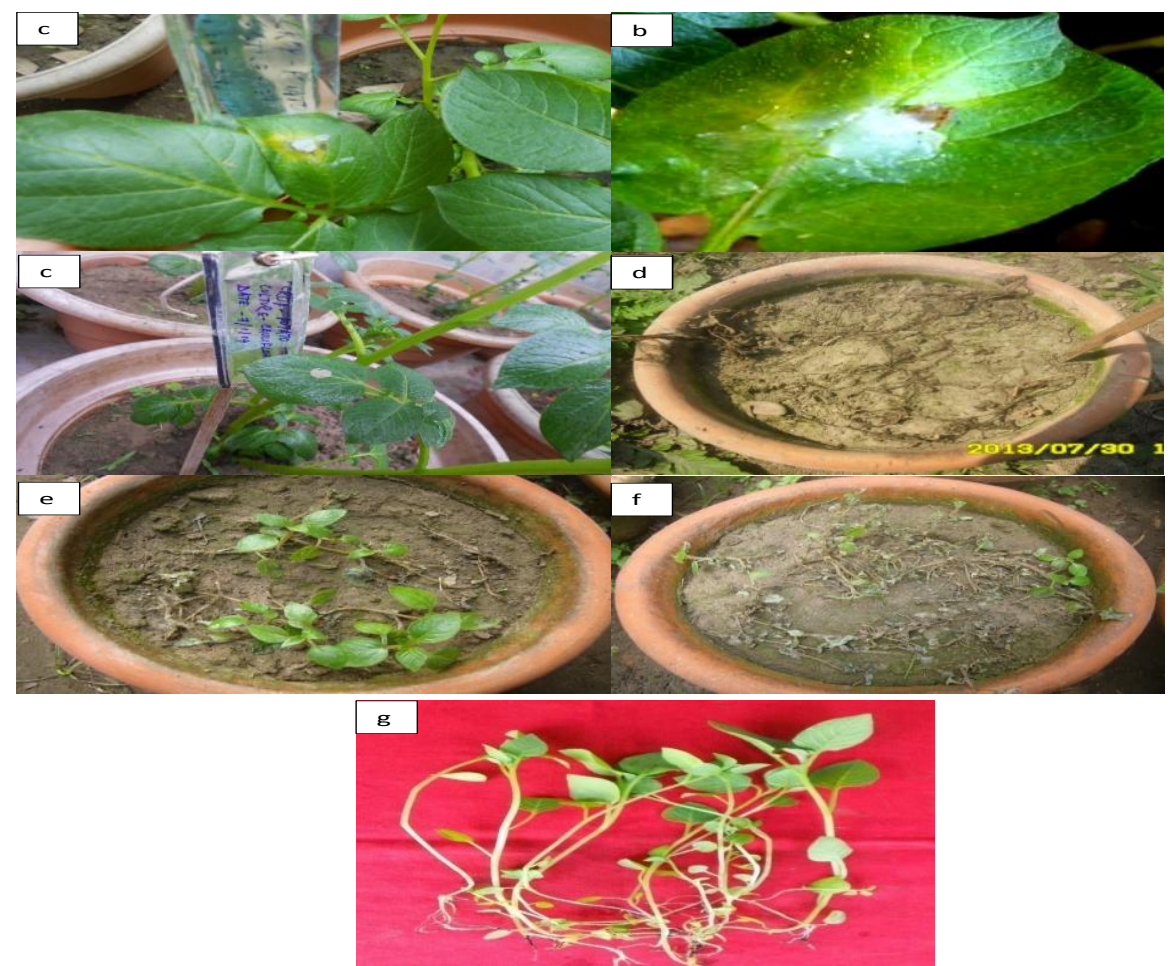

(a: Pathogenicity test of RS-9 on potato, b: Infection of RS-4 on potato leaves, c: Infection of RS-5 on potato leaves, d: Infection of RS-6 on potato leaves, e: Infection of RS-8 on potato on hypocotyl, f: Infection of RS-9 on potato on hypocotyl, g: Healthy potato plant in control) 


\section{Pathogenicity test on leaves}

Results of cross inoculation of all the isolates of $R$. solani showed different reaction on the leaves of five different crops (rice, maize, green gram, cabbage and potato). Disease severity score varied from 0 to 5 . Highest disease severity score of 5 was recorded on cabbage for all the 10 isolates of $R$. solani. All the infected seedling were found dead after 15 days of its germination. Rice isolate RS-1 caused infection to all the tested crops (Plate 4d) except potato (Table 2). Maximum disease severity score of 5 was recorded when RS-1 tested against the leaves of cabbage whereas disease severity score 4 was recorded on rice leaves and disease severity score 3 was recorded for maize and green gram (Plate 4f). All the 10 isolates of $R$. solani caused infection on leaves of maize and cabbage. Isolates RS-1, RS-2, RS-3, RS-7 and RS-8 cause infection on all the tested crops except potato leaves. Isolates RS-4, RS-5, RS-6, RS9 and RS-10 did not cause infection on maize. RS-9 and RS-10 did not cause infection on rice (Table 2). Isolate RS-8 did not cause infection on green gram showing healthy after its inoculation (Plate 4e), whereas isolate RS3 caused typical symptom of Rhizoctonia infection on green gram (Plate 4f). RS-4 caused severe infection on inoculation to green gram which also developed aerial mycelium (Plate 4g). Isolate RS-8 on inoculation to rice developed typical symptom of Rhizoctonia infection (Plate 5a). Similarly, RS-5 and RS-7 also caused infection with disease severity score of 4 (Plate $5 c$ and d). No infection was recorded in the control pot (Plate 5e). Results on pathogenicity of potato showed that RS-9 did not cause any infection of potato leaves with disease severity score 0 (Plate 6a) whereas, RS-4, RS-5 and Rs-6 caused infection on potato leaves with disease severity score of 3 (Plate $6 c, d$ and e). On the other hand, RS-7, RS-8 and RS-9 on inoculation to soil cause infection on hypocotyls of potato with disease severity score of 4, 4 and 5 respectively (Plate $6 \mathrm{~g}$, e and f).

\section{Cultural variability}

All the isolates of $R$. solani showed great diversity in terms of colony colour, growth pattern and radial growth. Based on colony pigmentation isolates were assigned to four (4) groups as white, grey, yellow and orange (Debbarma and Dutta, 2015). Six isolates of $R$. solani while studying variability was observed as light brown, five isolates were found yellowish brown, four isolates were whitish brown in colour, six isolates were dark brown and four isolates were very pale brown (Lal and Kandhari, 2009). On the basis of growth pattern, the isolates were categorized into three groups: abundant, moderate and slight. Similarly, Burpee et al., (1980) had also grouped the growth pattern of $R$. solani in to same three groups, viz., abundant, moderate and scarce.

\section{Morphological variability}

Compound microscope studies revealed that all the 10 isolates of $R$. solani characteristically having hyphal branching at right angle, septate with lateral branches constricted at the junction. A similar report was also given by Lal and Kandhari (2009) while studying on the variability of 25 isolates of $R$. solani. In their compound microscope studies they found that all the 25 isolates of $R$. solani under study was characteristically having hyphal branching at right angle, constriction at the point of branching of the mycelium and presence of a septum near the branching junction. Hyphal width of all the 10 isolates of $R$. solani ranged from $5.6 \mu \mathrm{m}$ to $7.2 \mu \mathrm{m}$. Based on the texture, sclerotia was classified in two groups viz., smooth and rough. Out of 24 isolates under study, 12 isolates were grouped in smooth category, and 
remaining 12 isolates in rough category (Hoa, 1994).

\section{Pathogenicity test}

Results of cross inoculation of all the ten (10) isolates of $R$. solani showed positive reaction to the hypocotyl of potato with severity score 4 (seedling growth is hampered, large lesions on the lower stem and roots). Highest disease severity score of 5 was recorded by RS- 1 to RS-6 and RS-9 and RS-10 was on cabbage. All the infected seedling were found dead after 15 days of its germination. RS-3, RS-5, RS-6, RS-9 and RS-10 could not able cause infection on maize showing disease severity score of 0 i.e., seedling well developed, no visible lesions on lower roots developed. Similarly, RS-6 to RS-10 did not cause infection on green gram but RS-1 to RS-5 cause severe infection with severity score of 5 causing total death of the seedlings. Similarly, results of cross inoculation of all the 10 isolates of $R$. solani showed different reaction on the leaves of five different crops (rice, maize, green gram, cabbage and potato). Disease severity score varied from 0 to 5 . Highest disease severity score of 5 was recorded on cabbage for all the 10 isolates of $R$. solani. All the infected seedling were found dead after 15 days of its germination. Rice isolate RS-1 caused infection to all the tested crops except potato. RS-4, RS-5, RS-6, RS-9 and RS-10 did not cause infection on maize. Similarly, RS-1 to RS-3 and RS-7 to RS-10 did not showed infection on potato leaves. Few reports in the literature have shown the virulence of AG-4 isolates on roots and aerial parts of plants, but it was low to moderate in latter tissues (Olaya et al., 1994).However, in another study, AG-4 failed to cause foliar symptoms but proved highly virulent on hypocotyls or roots of dry bean and soybean (Muyolo et al., 1993). Disease severity was lowest in rice seedlings inoculated with isolates R3 and R5, and highest in those inoculated with isolates R9 and R11 (Basu et al., 2004). Dath (1985) also reported that rice isolates of $R$. solani with larger sclerotia were more virulent. Li et al., (1998) have also described the pathogenicity of AG-4 isolates on sheaths of maize. In an study Dutta et al., (2015) reported the pathogenicity

In conclusion a great diversity in the genus Rhizoctonia has been observed culturally, morphologically and genetically along with the wide host range of the genus. Rhizoctonia represents a diverse group of fungi that differs in many significant features. Identification of Rhizoctonia isolates to some taxonomic level is of utmost importance for studying their epidemiology and control in different cropping systems. Therefore, knowledge of the specific Rhizoctonia spp. and anastomosis groups and their pathogenicity is important. In the present studies pathogenic variability of different isolates has been observed. All the isolates did not found have the ability of infects all the tested host. Even at disease score variability has been observed. So, we suggests, further studies on anastomosis classification of multinucleate and binucleate species of Rhizoctonia.

\section{References}

Basu, A., M.Podderand Sengupta, P.K. 2004. Variability and anastomosis among the rice isolates of $R$. solani. Indian Phytopathology. 57: 7.

Burpee, L.L.,P.L. Sander and Sherwood, R.T.1980. Anastomosis group among isolates of Ceratobasidiumcor nigerum (Bourd) Rogers and related fungi. Mycologia72: 689-701.

Das, B.C., Khairuzzaman, Islam, N and Dutta, P. 1997. Biological seed treatment for management of sheath blight of rice. In: Proceedings of International Symposium on Rainfed Rice 
Production Strategy for Twenty First Century. Organized by Assam Agricultural University, Jorhat,25-27 Nov, 1997. pp. 315-319.

Dath, P.A., 1985. A better criterion in rating the reaction of rice cultivars against sheath blight. Indian Phytopathology. 38: 678-682.

Debbarma, M., and Dutta, P. 2015. Cultural and morphological variability in Rhizoctonia solaniIsolates of different hosts of Assam. Indian Journal of Applied Sciences.5(2): 61-67.

Dutta, P.,and Das, B. C. 2002. Management of collar rot of tomato by Trichoderma sppand chemicals. Indian Phytopathology, 55(2): 235-237.

Dutta, P., and Das, B.C.1999. Effect of seed pelleting and soil application of Trichoderma harzianum in the management of stem rot of soybean. Journal of Mycology andPlant Pathology,29(3): 317-322.

Gonzalez, D., D.E. Carling, S.Kuninga, R.Vilgalys, and Cubeta, M.A. 2001. Ribosomal DNA systematic of Ceratobasidium and Thanatephorus with Rhizoctonia anamorphs. Mycology,93: 1138-1150.

Gonzalez, D.; M.A.Cubeta and Vilgalys, R. 2006. Phylogeneticutility of indels within ribosomal DNA and b-tubulin sequences from fungi in the Rhizoctonia solani species complex. Molecular

PhylogeneticEvoutionary.40: 459-470

Hoa, T.T.C. (1994). Characterization and pathogenicity of Rhizoctonia solani Kuhn isolates from different rice zone and management of sheath blight of rice. Ph.D Thesis, Indian Agricultural Research Institute,New Delhi-12, p. 122.

Kuninga, S., T.Natsuaki, T.Takeuchi, and Yokosawa, R. 1997. Sequence variation of the rDNA ITS regions within and between anastomosis groups in Rhizoctonia solani. Current Genetics.32: 237-243.

Lal, M. and Kandhari, J. 2009. Cultural and morphological variability in Rhizoctonia solani isolates causing sheath blight of rice. Journal of Mycology and Plant Pathology.39(1):77-81.

Li, H.R., B.C. Wu and Yan, S.Q. (1998). Aetiology of Rhizoctonia in sheath blight of maize in Sichuan. Plant Pathology 47: 16-21.

Muyolo, N.G., P.E.Lipps and Schmitthenner, A.F. 1993. Anastomosis grouping and variation in virulence among isolates of Rhizoctonia solani associated with dry bean and soybean in Ohio and Zaire. Phytopathoogy. 83: 438-444.

Olaya, G. and Abawi, G.S. 1994. Characterization of Rhizoctonia solani and binucleate Rhizoctonia species causing foliar blight and root rot on table beets in New York state. Pl. Dis. 78: 800-804. Sharon, M.; Kuninaga, S.; Hyakumachi, M. and Sneh, B. (2006). The advancing identification and classification of Rhizoctonia spp. using molecular and biotechnological methods compared with the classical anastomosis grouping. Mycoscience. 47: 299-316.

Sharon, M., S.Kuninaga, M.Hyakumachi, and Sneh, B. 2006. The advancing identification and classification of Rhizoctonia spp. using molecular and biotechnological methods compared with the classical anastomosis grouping. Mycoscience.47: 299-316.

Sharon, M., S.Kuninaga, M. Hyakumachi, S.Naito and Sneh, B. 2008. Classification of Rhizoctonia spp. using rDNA-ITS sequence analysis supports the genetic basis of the classical anastomosis grouping. Mycoscience.49(2): 93-114. 
Sneh, B. S.Jabaji-Hare, S. Neate, and Dijst, G. 1996. Rhizoctonia species: Taxonomy, Molecular Biology, Ecology, Pathology, and Control, Kluwer Acad. Publishers, Dordrecht, The Netherlands, p. 578.

Zhou, Q., K. Chang, Hwang, S.S.E.Strelkov,
B.D. Gossen and Chen, Y. 2009. Pathogenicity and genetic diversity of Rhizoctonia solani isolates from lupin and other crops in Alberta, Canada. Canadian Journal of Plant Pathology. 31: 340-343.

\section{How to cite this article:}

Manashi Debbarma and Pranab Dutta. 2021. Pathogenic Variability of Isolates of Rhizoctonia solani of Different Agricultural Crops. Int.J.Curr.Microbiol.App.Sci. 10(01): 765-776. doi: https://doi.org/10.20546/ijcmas.2021.1001.094 